\title{
Absence of skew scattering in two-dimensional systems: Testing the origins of the anomalous Hall effect
}

\author{
Mario F. Borunda, ${ }^{1}$ Tamara S. Nunner, ${ }^{2}$ Thomas Lück, ${ }^{2}$ N. A. Sinitsyn, ${ }^{3,1}$ Carsten \\ Timm, ${ }^{4}$ J. Wunderlich, ${ }^{5}$ T. Jungwirth,${ }^{6,7}$ A. H. MacDonald, ${ }^{8}$ and Jairo Sinova ${ }^{1}$ \\ ${ }^{1}$ Department of Physics, Texas A\&M University, College Station, TX 77843-4242, USA \\ ${ }^{2}$ Institut für Theoretische Physik, Freie Universität Berlin, Arnimallee 14, 14195 Berlin, Germany \\ ${ }^{3}$ CNLS/CCS-3, Los Alamos National Laboratory, Los Alamos, NM 87545, USA \\ ${ }^{4}$ Department of Physics and Astronomy, University of Kansas, Lawrence, KS 66045, USA \\ ${ }^{5}$ Hitachi Cambridge Laboratory, Cambridge CB3 OHE, UK \\ ${ }^{6}$ Institute of Physics ASCR, Cukrovarnická 10, 162 53 Praha 6, Czech Republic \\ ${ }^{7}$ School of Physics and Astronomy, University of Nottingham, Nottingham NGr 2RD, UK \\ ${ }^{8}$ Department of Physics, University of Texas at Austin, Austin TX 78712-1081, USA
}

(Dated: February 12, 2007)

\begin{abstract}
We study the anomalous Hall conductivity in spin-polarized, asymmetrically confined twodimensional electron and hole systems, focusing on skew-scattering contributions to the transport. We find that the skew scattering, principally responsible for the extrinsic contribution to the anomalous Hall effect, vanishes for the two-dimensional electron system if both chiral Rashba subbands are partially occupied, and vanishes always for the two-dimensional hole gas studied here, regardless of the band filling. Our prediction can be tested with the proposed coplanar two-dimensional electron/hole gas device and can be used as a benchmark to understand the crossover from the intrisic to the extrinsic anomalous Hall effect.
\end{abstract}

PACS numbers: 72.15.Eb,72.20.Dp,72.25.-b

Introduction.-The observed Hall resistance of a magnetic film contains the ordinary Hall response to the external magnetic field and the anomalous Hall response to the internal magnetization. Although the anomalous Hall effect (AHE) has been used for decades as a basic characterization tool for ferromagnets, its origin is still being debated, also in the context of a closely related novel phenomenon, the spin Hall effect [1, 2, 3, 4 4 . Three mechanisms giving rise to AHE conductivity have been identified: (1) an intrinsic mechanism based solely on the topological properties of the Bloch states originating from the spin-orbit-coupled electronic structure [5], (2) a skew-scattering mechanism originating from the asymmetry of the scattering rate [6], and (3) a side-jump contribution, which semiclassically is viewed as a sidestep-type of scattering and contributes to a net current perpendicular to the initial momentum [7].

Recent experimental and theoretical studies of transition-metal ferromagnets and of less conventional systems, such as diluted magnetic semiconductors, oxide and spinel ferromagnets, etc., have collected numerous examples of the intrinsic AHE and of the transition to the extrinsic AHE dominated by disorder scattering [8]. The unambiguous determination of the origin of the AHE in these experimental systems is hindered, in part, by their complex band structures, which has motivated studies of simpler model Hamiltonians, such as the two-dimensional (2D) Rashba and Dirac band models [9, 10, 11, 12, 13]. Attempts to describe all the contributions to the AHE within the same framework have yielded farraginous results, however. So far a rigorous connection of the more intuitive semiclassical transport treatment with the more systematic diagramatic treatment, providing a clear-cut interpretation of the intrinsic, skew, and side-jump AHE terms, has only been demonstrated for the Dirac Hamiltonian model [14].

In this Letter we calculate the transport coefficients in these two complementary approaches for asymmetrically confined 2D electron and hole gases in the presence of spin-independent disorder, finding perfect agreement. The motivation for the study of these systems is threefold: First, they can be represented by simple spin-orbit-coupled bands, which, similar to the Dirac Hamiltonian model, allows us to unambiguously identify the individual AHE contributions. Second, the extrinsic skew-scattering term vanishes for a two-subband occupation in the case of the Rashba 2D electron gas and for any band occupation for the studied 2D hole gas. This provides a clean test of the intrinsic AHE mechanism and of the transition between the intrinsic and skew-scattering-dominated AHE. Finally, we propose a $2 \mathrm{D}$ electron gas/2D hole gas coplanar magneto-optical device in which the unique AHE phenomenology found in our theoretical models can be systematically explored experimentally.

Model Hamiltonians.-We study the following 2D model Hamiltonians:

$$
H=\frac{\hbar^{2} k^{2}}{2 m} \boldsymbol{\sigma}_{0}+i \alpha_{n}\left(\boldsymbol{\sigma}_{+} k_{-}^{n}-\boldsymbol{\sigma}_{-} k_{+}^{n}\right)-h \boldsymbol{\sigma}_{z}+V(\mathbf{r}) \boldsymbol{\sigma}_{0}
$$

with $m$ being the effective in-plane mass, $\boldsymbol{\sigma}_{i}$ the $2 \times 2$ Pauli matrices, $k_{ \pm}=k_{x} \pm i k_{y}, h$ the exchange field, $\alpha_{n}$ the spin-orbit coupling parameter, and $V(\mathbf{r})$ a spinindependent disorder potential. The exponent $n=1$ (3) 
describes a 2D electron (hole) gas [15]. The eigenenergies of the clean system are $E_{ \pm}=\hbar^{2} k^{2} / 2 m \pm \sqrt{h^{2}+\left(\alpha_{n} k^{n}\right)^{2}}$. The eigenvectors in the clean system take the form $\left|\Psi_{\mathbf{k}}^{ \pm}\right\rangle=\exp (i \mathbf{k} \cdot \mathbf{r})\left|u_{\mathbf{k}}^{ \pm}\right\rangle$with $\mathbf{k}=k(\cos \phi, \sin \phi)$ and

$$
\left|u_{\mathbf{k}}^{ \pm}\right\rangle=\frac{1}{\sqrt{2 \lambda}}\left(\begin{array}{c} 
\pm i e^{-n i \phi} \sqrt{\lambda \pm h} \pm \\
\sqrt{\lambda \mp h}
\end{array}\right)
$$

where $\lambda=\sqrt{h^{2}+\left(\alpha_{n} k^{n}\right)^{2}}$. We now define $k_{ \pm}(E)$ as the wave number for the \pm band at a given energy $E$ and define $\lambda_{ \pm} \equiv \lambda\left(k_{ \pm}\right)$. If $E$ is not specified, it is assumed to be the Fermi energy. We consider the model of randomly located $\delta$-function scatterers, $V(\mathbf{r})=\sum_{i} V_{i} \delta\left(\mathbf{r}-\mathbf{R}_{i}\right)$ with $\mathbf{R}_{i}$ random and disorder averages satisfying $\left\langle V_{i}\right\rangle_{\text {dis }}=0$, $\left\langle V_{i}^{2}\right\rangle_{\text {dis }}=V_{0}^{2} \neq 0$, and $\left\langle V_{i}^{3}\right\rangle_{\text {dis }}=V_{1}^{3} \neq 0$. This model is different from the standard white-noise disorder with $\left\langle\left|V_{\mathbf{k}^{\prime} \mathbf{k}}^{0}\right|^{2}\right\rangle_{\text {dis }}=n_{i} V_{0}^{2}$, where $n_{i}$ is the impurity concentration and other correlators are either zero or related to this correlator by Wick's theorem. The deviation from white noise in our model is quantified by $V_{1} \neq 0$, and is necessary to capture part of the skew-scattering contribution to the AHE.

Semiclassical approach.-We sketch here the semiclassical procedure used in the calculation, for further details we refer to Ref. 14. The multi-band Boltzmann equation in a weak electric field $\mathbf{E}$ is given by

$$
\frac{\partial f_{l}}{\partial t}+e \mathbf{E} \cdot \mathbf{v}_{l} \frac{d f_{l}}{d \epsilon}=I[f]_{\mathrm{coll}}
$$

where $l=(\mathbf{k}, \mu), \mu= \pm$ is the subband index, and $I[f]_{\text {coll }}=-\sum_{\mu^{\prime}} \int d^{2} \mathbf{k}^{\prime} /(2 \pi)^{2} \omega_{l l^{\prime}}\left(f_{l}-f_{l^{\prime}}\right)$ is the impurity collision integral. The distribution function $f_{l}$ is the sum of the equilibrium function and a correction, $f_{l}=f_{l}^{0}+g_{l}$. The scattering rates $\omega_{l l^{\prime}}$ are related to the T-matrix elements through $\omega_{l l^{\prime}}=2 \pi / \hbar\left|T_{l^{\prime} l}\right|^{2} \delta\left(\epsilon_{l^{\prime}}-\epsilon_{l}\right)$, where $T_{l^{\prime} l}=\left\langle l^{\prime}|V| \psi_{l}\right\rangle$, and $\left|\psi_{l}\right\rangle$ are eigenstates of the complete Hamiltonian, and $|l\rangle \equiv\left|\Psi_{\mathbf{k}}^{\mu}\right\rangle$ of the disorderfree Hamiltonian.

Skew scattering.- Skew scattering appears in the Boltzmann equation through the asymmetric part of the scattering rate, i.e., $\omega_{l l^{\prime}} \neq \omega_{l^{\prime} l}[6]$. The scattering rates to second and third order in disorder strength are given by $\omega_{l l^{\prime}}=\omega_{l l^{\prime}}^{(2)}+\omega_{l l^{\prime}}^{(3)}+\cdots$, where $\omega_{l l^{\prime}}^{(2)}=2 \pi / \hbar\left\langle\left|V_{l l^{\prime}}\right|^{2}\right\rangle_{\text {dis }} \delta\left(\epsilon_{l}-\right.$ $\left.\epsilon_{l^{\prime}}\right)$ is symmetric. Here $V_{l^{\prime} l}=\left\langle l^{\prime}|V| l\right\rangle$. We break up the third-order contribution into symmetric and antisymmetric parts. We ignore the first, since only the second gives rise to skew scattering. This antisymmetric term is given by [14]

$\omega_{l l^{\prime}}^{(3 a)}=-\frac{(2 \pi)^{2}}{\hbar} \sum_{l^{\prime \prime}} \delta\left(\epsilon_{l}-\epsilon_{l^{\prime \prime}}\right) \operatorname{Im}\left\langle V_{l l^{\prime}} V_{l^{\prime} l^{\prime \prime}} V_{l^{\prime \prime} l}\right\rangle_{\mathrm{dis}} \delta\left(\epsilon_{l}-\epsilon_{l^{\prime}}\right)$

The solution of the Boltzmann equation (3) is found by first looking at the deviation of the distribution function from equilibrium [14],

$$
g_{l}=-\frac{\partial f_{\mu}^{0}}{\partial \epsilon} e E\left|v_{\mu}\right|\left(A_{\mu} \cos \phi+B_{\mu} \sin \phi\right) .
$$

Assuming that the transverse conductivity is much smaller than the longitudinal one $\left(A_{\mu} \gg B_{\mu}\right)$ and substituting Eq. (5) into Eq. (3) one finds $A_{\mu}=\tau_{\mu}^{\|}$and $B_{\mu}=\left(\tau_{\mu}^{\|}\right)^{2} / \tau_{\mu}^{\perp}$, where

$$
\begin{aligned}
\frac{1}{\tau_{\mu}^{\|}} & =\sum_{\mu^{\prime}} \int \frac{d^{2} \mathbf{k}^{\prime}}{(2 \pi)^{2}} \omega_{l l^{\prime}}\left[1-\frac{\left|v_{l^{\prime}}\right|}{\left|v_{l}\right|} \cos \left(\phi-\phi^{\prime}\right)\right], \\
\frac{1}{\tau_{\mu}^{\perp}} & =\sum_{\mu^{\prime}} \int \frac{d^{2} \mathbf{k}^{\prime}}{(2 \pi)^{2}} \omega_{l l^{\prime}} \frac{\left|v_{l^{\prime}}\right|}{\left|v_{l}\right|} \sin \left(\phi-\phi^{\prime}\right) .
\end{aligned}
$$

For symmetric Fermi surfaces, the skew-scattering contribution to the conductivity tensor at zero temperature can now be expressed using the scattering times,

$$
\sigma_{x x}=\frac{e^{2}}{4 \pi \hbar} \sum_{\mu} \tau_{\mu}^{\|} v_{F, \mu} k_{\mu}, \sigma_{x y}^{\text {skew }}=\frac{e^{2}}{4 \pi \hbar} \sum_{\mu} \frac{\left(\tau_{\mu}^{\|}\right)^{2}}{\tau_{\mu}^{\perp}} v_{F, \mu} k_{\mu} .
$$

The calculation of $\left(\tau_{\mu}^{\|}\right)^{-1}$ and $\left(\tau_{\mu}^{\perp}\right)^{-1}$ uses the matrix elements of Eq. (4). To simplify the notation we define

$$
\left\langle\mu \mu^{\prime}, \mu^{\prime} \mu^{\prime \prime}, \mu^{\prime \prime} \mu\right\rangle \equiv \operatorname{Im} \int_{0}^{2 \pi} d \phi^{\prime \prime}\left\langle u_{\mathbf{k}}^{\mu} \mid u_{\mathbf{k}^{\prime}}^{\mu^{\prime}}\right\rangle\left\langle u_{\mathbf{k}^{\prime}}^{\mu^{\prime}} \mid u_{\mathbf{k}^{\prime \prime}}^{\mu^{\prime \prime}}\right\rangle\left\langle u_{\mathbf{k}^{\prime \prime}}^{\mu^{\prime \prime}} \mid u_{\mathbf{k}}^{\mu}\right\rangle,
$$

where all momenta are taken on the Fermi surface. Note that in Eq. (9) the magnitude of $\mathbf{k}^{\prime \prime}$ can be different from that of $\mathbf{k}^{\prime}$ or $\mathbf{k}$ since the Fermi momenta of different bands do not coincide.

The matrix elements appearing in Eq. (9) can be calculated directly from the basis functions, yielding

$$
\left\langle\mu \mu^{\prime}, \mu^{\prime} \mu^{\prime \prime}, \mu^{\prime \prime} \mu\right\rangle=-\frac{h \pi \alpha_{n}^{2} k_{\mu}^{n} k_{\mu^{\prime}}^{n}}{2 \lambda_{\mu} \lambda_{\mu^{\prime}} \lambda_{\mu^{\prime \prime}}} \sin \left(n \phi-n \phi^{\prime}\right)
$$

from which we obtain

$$
\omega_{l l^{\prime}}^{(3 a)}=-\frac{1}{\hbar} n_{i} V_{1}^{3} \delta\left(\epsilon_{l}-\epsilon_{l^{\prime}}\right) \sum_{\mu^{\prime \prime}} \nu^{\mu^{\prime \prime}}\left\langle\mu \mu^{\prime}, \mu^{\prime} \mu^{\prime \prime}, \mu^{\prime \prime} \mu\right\rangle,
$$

where $\nu^{ \pm}$is related to the density of states of each band at the Fermi energy, $\left(\nu^{ \pm}\right)^{-1}=\hbar^{2} / m \pm n\left(\alpha_{n} k_{ \pm}^{n-1}\right)^{2} / \lambda_{ \pm}$. The symmetric part of the scattering rates to second order in the disorder potential is given by $\omega_{l l^{\prime}}^{(2)}=$ $2 \pi / \hbar n_{i} V_{0}^{2}\left|\left\langle u_{k}^{\mu} \mid u_{k^{\prime}}^{\mu^{\prime}}\right\rangle\right|^{2} \delta\left(\epsilon_{l}-\epsilon_{l^{\prime}}\right)$. The relaxation times are found by inserting this into Eq. (6) and Eq. (11) into Eq. (7). For $n=1$, i.e., for the $2 \mathrm{D}$ electron gas, the relaxation rates are then

$$
\begin{aligned}
\frac{1}{\tau_{\mu}^{\|}}= & \frac{1}{\hbar} n_{i} V_{0}^{2}\left[\frac{\nu^{\mu}}{\lambda_{\mu}}\left(\frac{h^{2}}{\lambda_{\mu}}+\frac{\alpha_{1}^{2} k_{\mu}^{2}}{4 \lambda_{+}}+\frac{\alpha_{1}^{2} k_{-}^{2}}{4 \lambda_{-}}\right)\right. \\
& \left.+\frac{\nu^{\bar{\mu}}}{2}\left(1-\frac{h^{2}}{\lambda_{-} \lambda_{+}}\right)\right] \\
\frac{1}{\tau_{\mu}^{\perp}}= & -\frac{n_{i} V_{1}^{3} h \alpha_{1}^{2} \nu^{\mu}}{8 \hbar \lambda_{\mu}}\left(\frac{k_{\mu}^{2}}{\lambda_{\mu}}-\frac{k_{\bar{\mu}}^{2}}{\lambda_{\bar{\mu}}}\right)\left(\frac{\nu^{\mu}}{\lambda_{\mu}}-\frac{\nu^{\bar{\mu}}}{\lambda_{\bar{\mu}}}\right)
\end{aligned}
$$




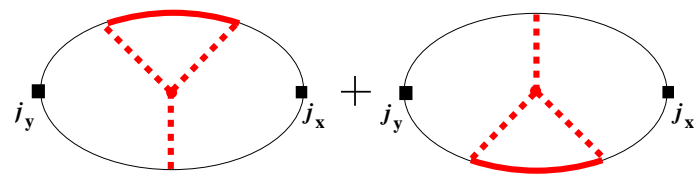

FIG. 1: (color online). Diagramatic representation of the skew-scattering contribution to $\sigma_{y x}$. Both current vertices, denoted by squares, are renormalized by ladder vertex corrections.

where $\bar{\mu} \equiv-\mu$. If both subbands are occupied, the last factor in Eq. (13) vanishes and there is no skew-scattering contribution. If only the majority subband is occupied $\left(E_{F}<h\right),\left(\tau_{\mu}^{\perp}\right)^{-1}$ is non-zero and skew scattering contributes. For the skew-scattering Hall conductivity and the longitudinal conductivity we obtain in this case

$$
\begin{aligned}
\sigma_{x x} & =\frac{e^{2}}{\pi \hbar n_{i} V_{0}^{2}}\left(\frac{\lambda_{-} k_{-}}{\nu^{-}}\right)^{2} \frac{1}{3 h^{2}+\lambda_{-}^{2}}, \\
\sigma_{x y}^{\text {skew }} & =-\frac{e^{2} V_{1}^{3}}{2 \pi \hbar n_{i} V_{0}^{4}} \frac{h \lambda_{-} \alpha_{1}^{2} k_{-}^{4}}{\nu_{-}\left(3 h^{2}+\lambda_{-}^{2}\right)^{2}} .
\end{aligned}
$$

If $n=3$, i.e., for the $2 \mathrm{D}$ hole gas, we obtain

$$
\begin{aligned}
\frac{1}{\tau_{\mu}^{\|}} & =\frac{1}{\hbar} n_{i} V_{0}^{2}\left[\frac{\nu^{\mu}}{2 \lambda_{\mu}^{2}}\left(\lambda_{\mu}^{2}+h^{2}\right)+\frac{\nu^{\bar{\mu}}\left(\lambda_{-} \lambda_{+}-h^{2}\right)}{2 \lambda_{-} \lambda_{+}}\right], \\
\frac{1}{\tau_{\mu}^{\perp}} & =0
\end{aligned}
$$

and skew scattering vanishes irrespective of band filling.

Microscopic approach.-Within the diagramatic Kubo formalism the skew-scattering contribution to the offdiagonal conductivity is obtained from the expression

$$
\sigma_{x y}^{I(a)}=\frac{e^{2} \hbar}{2 \pi V} \sum_{k} \operatorname{Tr}\left[v_{x} G_{k}^{R}\left(E_{F}\right) v_{y} G_{k}^{A}\left(E_{F}\right)\right],
$$

where the bare velocity vertex factors in the linear-in-k Rashba model are given by

$$
v_{x}=\frac{\hbar k_{x}}{m} \boldsymbol{\sigma}_{0}-\frac{\alpha_{1}}{\hbar} \boldsymbol{\sigma}_{y}, \quad v_{y}=\frac{\hbar k_{y}}{m} \boldsymbol{\sigma}_{0}+\frac{\alpha_{1}}{\hbar} \boldsymbol{\sigma}_{x} .
$$

As shown in a previous study [14], the skew-scattering contribution proportional to $V_{1}^{3} /\left(n_{i} V_{0}^{4}\right)$ corresponds to the diagrams shown in Fig. 1, where the current vertices $j_{x}, j_{y}$ on both sides are the bare velocities $v_{x}, v_{y}$ renormalized by ladder vertex corrections. Only the skew-scattering diagrams with a single third-order vertex, shown in Fig. 1, contribute to order $V_{1}^{3} /\left(n_{i} V_{0}^{4}\right)$. All other terms from a ladder-type summation of third-order vertices are smaller because they are either not of the order $1 / n_{i}$ or of higher order in $V_{1} / V_{0}$. The sum of the skew-scattering vertices (i.e., the bold/red part of Fig. 1) gives

$$
\frac{i}{4} n_{i} V_{1}^{3} h\left(\frac{\nu_{-}}{\lambda_{-}}-\frac{\nu_{+}}{\lambda_{+}}\right)\left(\boldsymbol{\sigma}_{0} \otimes \boldsymbol{\sigma}_{z}-\boldsymbol{\sigma}_{z} \otimes \boldsymbol{\sigma}_{0}\right) .
$$

In the linear Rashba model we find $\nu_{+} / \lambda_{+}=\nu_{-} / \lambda_{-}$, implying that skew scattering vanishes if both subbands are occupied. In the case that only one subband is occupied the evaluation of Fig. 1 to order $V_{1}^{3} /\left(n_{i} V_{0}^{4}\right)$ yields exactly the same expression for $\sigma_{x y}^{\text {skew }}$ as in the semiclassical Eq. (15)). The only effect of the ladder vertex corrections is to renormalize each bare velocity by a factor of $2\left(h^{2}+\lambda_{-}^{2}\right) /\left(3 h^{2}+\lambda_{-}^{2}\right)$ which reduces to a factor of 1 in the limit of small $\alpha_{1} k_{F}$ and to a factor of 2 in the limit of small $h$.

For the 2D hole-gas model Hamiltonian (1) with $n=3$ the bare velocity vertex factors are

$$
v_{x, y}=\frac{\hbar k_{x, y}}{m} \boldsymbol{\sigma}_{0}-\frac{6 \alpha_{3}}{\hbar} k_{x} k_{y} \boldsymbol{\sigma}_{y, x} \pm \frac{3 \alpha_{3}}{\hbar}\left(k_{x}^{2}-k_{y}^{2}\right) \boldsymbol{\sigma}_{x, y} .
$$

Here the vertex corrections disappear because integrals of the type $\sum_{k} G_{k}^{R} v_{x, y} G_{k}^{A}=0$ vanish. This implies the absence of skew scattering for any subband filling [16], consistent with the semiclassical result. We note that the same consistency between semiclassical and microscopic quantum theory calculations for the studied 2D models is also obtained for the intrinsic and side-jump terms similar to the results in the graphene model [14]; the longer details of those calculations will be shown elsewhere and are in general agreement with Ref. 12 .

The abscence of the skew scattering is akin but not equivalent to the results of spin-Hall-effect calculations in 2D systems [17]. For the Rashba 2D electron gas the disappearance of the DC spin Hall conductivity is guaranteed by sum rules that relate the spin current to the dynamics of the induced spin polarization [18, 19]. In the case of a charge current no similar sum rule is known. As we have shown, the skew-scattering contribution in fact becomes finite when the minority band is depleated. The vanishing of the Hall conductivity in the Rashba $2 \mathrm{D}$ electron gas for $E_{F}>h$ is attributed to the simplicity of the Hamiltonian. In particular the relation $\nu_{+} / \lambda_{+}=\nu_{-} / \lambda_{-}$ does not hold generally beyond the case of the linear-in$\mathbf{k}$ Rashba coupling. The abscence of skew scattering in the 2D hole system has a different origin: Due to the cubic dependence of spin-orbit coupling on momentum, the matrix elements, Eq. (10), in the antisymmetric part of the collision term behave like $\sin \left(3 \phi-3 \phi^{\prime}\right)$. Together with the $\sin \left(\phi-\phi^{\prime}\right)$ dependence of the velocity factor in Eq. (7), this makes the integral over $\mathbf{k}^{\prime}$ vanish.

Our results predict that the AHE in 2D electron and hole systems can be dominated by contributions independent of the impurity concentration, for which the anomalous Hall resistance is $\propto \sigma_{x x}^{-2}$. We also predict that in the Rashba 2D electron gas with only one subband occupied the extrinsic skew-scattering contribution, leading to anomalous Hall resistance proportional to $\sigma_{x x}^{-1}$, is non-zero. Note that this term has not been identified in previous works that considered only white-noise disorder 9, 10, 11, 12, 13]. Since its corresponding conductivity contribution is inversely proportional to the impurity 

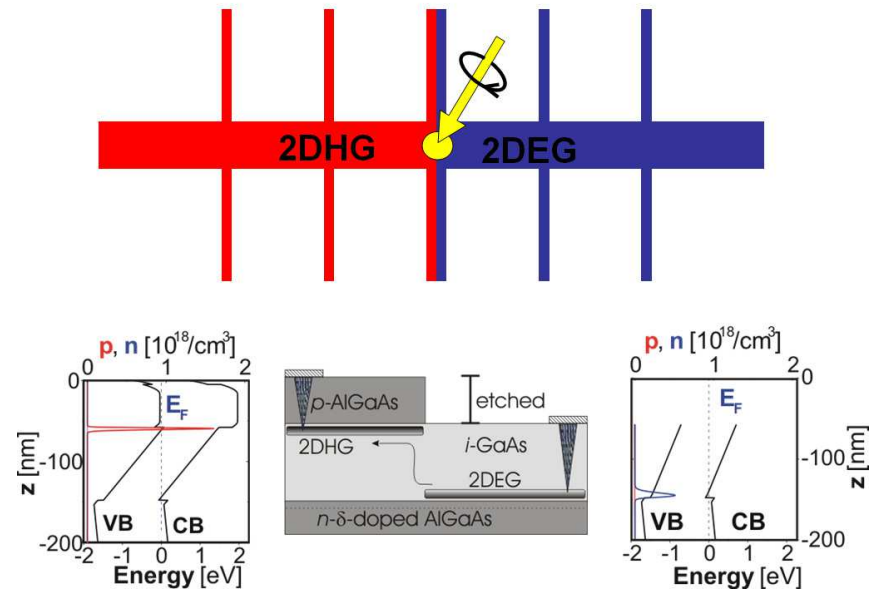

FIG. 2: (color online). Top panel: Top-view schematics of the Hall bar with coplanar 2D hole and electron gases. Spinpolarized carriers are generated by shining circularly polarized light on the $\mathrm{p}-\mathrm{n}$ junction. Center bottom panel: Cross section of the heterostructure containing p-type and n-type AlGaAs/GaAs single junctions. The left band diagram corresponds to the unetched part of the wafer with the $2 \mathrm{D}$ hole gas, the right band diagram shows the $2 \mathrm{D}$ electron gas in the etched section of the wafer.

concentration, the skew-scattering mechanism can dominate in clean samples.

Proposed experimental setup.-The unique phenomenology of the AHE in the studied 2D systems, in particular the sudden disappearance of skew scattering when the Fermi level crosses the depletion point of the minority 2D Rashba band, represents an opportunity for a clean test of the presence of intrinsic and extrinsic sources of the AHE and of the transition between these two regimes. In the absence of $2 \mathrm{D}$ ferromagnetic system with Rashba like spin-orbit interatciton, we proposed an experimental setup for this test as shown in Fig. 22 The device is based on a AlGaAs/GaAs heterostructure containing a coplanar $2 \mathrm{D}$ hole gas $/ 2 \mathrm{D}$ electron gas p-n junction. The cross section of the heterostructure and corresponding band diagrams are shown in the lower panels of Fig. 2 (for more details see Ref. 3 ). Under a forward bias the junction was successfully utilized as a light-emitting-diode spin detector for the spin Hall effect [3]. Here we propose to operate the junction in the reverse-bias mode, while shining monochromatic, circularly polarized light of tuneable wavelength on the p-n junction. The photogenerated spin-polarized holes and electrons will propagate in opposite directions through the respective $2 \mathrm{D}$ hole and electron channels. The longitudinal voltage and the generated anomalous Hall voltage can be detected by the successive sets of Hall probes, as shown in the upper panel of Fig. 2, For the 2D electron gas the macroscopic spin diffusion length allows to use standard lithography for defining the Hall probes. Surface or back gates in close proximity to the $2 \mathrm{D}$ electron system can be used to modify the effective 2D confinements, carrier density, and spin-orbit coupling in order to control the transition between the intrinsic and extrinsic AHE regimes. The exploration of the AHE in the 2D hole gas is more challenging due to the expected sub-micron spin diffusion length in this system but may still be feasible in the proposed experimental setup.

Fruitful discussions with S. Onoda and N. Nagaosa are gratefully acknowledged. This work was supported by ONR under Grant No. onr-n000140610122, by the NSF under Grants No. DMR-0547875 and No. PHY99-07949, by the SRC-NRI (SWAN), by EU Grant IST-015728, by EPSRC Grant GR/S81407/01, by GACR and AVCR Grants 202/05/0575, FON/06/E002, AV0Z1010052, and LC510, by the DOE under Grant No. DE-AC5206NA25396, and by the Univ. of Kansas General Research Fund allocation No. 2302015. Jairo Sinova is a Cottrell Scholar of Research Corporation.

[1] M. I. Dyakonov and V. I. Perel, JETP 467 (1971); S. Murakami, N. Nagaosa, and S.-C. Zhang, Science 301, 1348 (2003); J. Sinova et al., Phys. Rev. Lett. 92, 126603 (2004).

[2] Y. K. Kato et al., Science 306, 1910 (2004).

[3] J. Wunderlich et al., Phys. Rev. Lett. 94, 047204 (2005).

[4] V. Sih et al., Nature Physics 1, 31 (2005).

[5] R. Karplus and J. M. Luttinger, Phys. Rev. 95, 1154 (1954).

[6] J. Smit, Physica 21, 877 (1955).

[7] L. Berger, Phys. Rev. B 2, 4559 (1970).

[8] J. Banhart and H. Ebert, Europhys. Lett. 32, 517 (1995); J. Ye et al., Phys. Rev. Lett. 83, 3737 (1999); T. Jungwirth, Q. Niu, and A. H. MacDonald, ibid. 88, 207208 (2002); Y. Yao et al., ibid. 92, 037204 (2004); Y. Taguchi et al., Science 291, 5513 (2001); W.-L. Lee et al., ibid. 303, 1647 (2004); J. Kötzler and W. Gil, Phys. Rev. B 72, 060412(R) (2005); B. C. Sales et al., ibid. 73, 224435 (2006); C. Zeng et al., Phys. Rev. Lett. 96, 037204 (2006); S. H. Chun et al., ibid. 98, 026601 (2007); J. Cumings et al., ibid. 96, 196404 (2006); T. Miyasato et al., cond-mat/0610324.

[9] V. K. Dugaev et al., Phys. Rev. B 71, 224423 (2005).

[10] N. A. Sinitsyn et al., Phys. Rev. B 72, 045346 (2005).

[11] S. Y. Liu and X. L. Lei, Phys. Rev. B 72, 195329 (2005).

[12] J. Inoue et al., Phys. Rev. Lett. 97, 046604 (2006).

[13] S. Onoda, N. Sugimoto, and N. Nagaosa, Phys. Rev. Lett. 97, 126602 (2006).

[14] N. A. Sinitsyn et al., Phys. Rev. B 75, 045315 (2007).

[15] R. Winkler, Spin-Orbit Coupling Effects in Two- Dimensional Electron and Hole Systems, Springer Tracts in Modern Physics, vol. 191 (Springer, Berlin, 2003).

[16] B. A. Bernevig and S.-C. Zhang, Phys. Rev. Lett. 95, 016801 (2005).

[17] J. Inoue, G. E. W. Bauer, and L. W. Molenkamp, Phys. Rev. B 70, 041303 (2004), cond-mat/0402442.

[18] A. A. Burkov, A. S. Nuñez, and A. H. MacDonald, Phys. Rev. B 70, 155308 (2004).

[19] O. Chalaev and D. Loss, Phys. Rev. B 71, 245318 (2004). 